\title{
Research on application of Compactly Supported Biorthogonal wavelet in image Matching
}

\author{
Zhou Fang ${ }^{1,}$, Yan Cao ${ }^{1, b}$, Li-Na Zhang ${ }^{2, c}$ \\ ${ }^{1}$ School of Mechatronic Engineering Xi'an Technological University Xi'an, China \\ ${ }^{2}$ Xi'an Modern Chemistry Research Institute, Xi'an, China \\ a phoenix20030696@qq.com, ${ }^{\mathrm{b}}$ jantonyz@163.com, ${ }^{\mathrm{c}} 502991275 @ q q . c o m$
}

Keywords: micro-displacement; compactly supported wavelet; image matching

\begin{abstract}
Image matching solves the identification of identical regions between images in a moving image sequence that is collected by a CCD camera. Through image feature region matching, micro-displacement and micro-angular displacement can be measured based on digital image processing. In the paper, a shape function is presented to process the feature regions, and a compactly supported biorthogonal wavelet filter is constructed to match the feature regions. Test results show that the approach can effectively identify the same feature regions between the images in the moving image sequence. Matching errors are also analyzed and the approach is proved to be feasible.
\end{abstract}

\section{Introduction}

The description and matching of feature regions in images is to recognize the tiny relative displacement between the adjacent images captured by a CCD camera. From the computation of displacement of image, the movement of the CCD camera can be got inversely. Through the preprocessing and edge extraction, the feature regions in the images can be described as a non-periodic curve function that is made of discrete edge points. Using the non-periodic curve function and multi-resolution analysis of Compactly Supported Biorthogonal Wavelet, it is possible to describe and match the feature regions and to calculate the displacement of the CCD camera [1].

\section{Discrete expression of a profile}

An edge of any closed area in an image is defined as $\Omega$, it is composed of a group of discrete points, i.e., $\left\{a_{n}\right\}_{n=1}^{M}$. The coordinates of the discrete points are $\left\{\left(\mathrm{x}_{\mathrm{n}}, \mathrm{y}_{\mathrm{n}}\right)\right\}$. In order to get the periodic function, if $\mathrm{a}_{M+i}=\mathrm{a}_{i}$, the closed curve can be approximated by a closed polygon. The feature of the polygon can be decided by the distance between each discrete point and its central point. A shape function [2] can be obtained using the orthonormal arc length. The function has the translation, scaling and rotation invariants.

\section{Feature matching of the shape function}

The feature function of the closed curve is a periodic function of its arc-lengths. The feature function is an oscillating and non-smooth function, it is efficient that using Compactly Supported Biorthogonal Wavelet to describe the feature function. ${ }^{X_{[0,1]}}$ is a square integrable space, the period of the square integrable space is $1 . \psi_{j k}^{p}(x)$ and $\varphi_{j k}^{p}(x)$ are basic functions of ${ }^{W_{j}}$ and ${ }^{V_{j}}$ where is the wavelet space and $V_{j}$ is the scale function space. For the function $f(x) \in X_{[0,1]},\left\{\left\langle f, \psi_{j k}^{p}\right\rangle\right]_{k=0}^{2^{j-1}}$ is the $j$ level of wavelet coefficient, $s_{k}^{j}=\left\langle f, \varphi_{j k}^{p}\right\rangle$, and ${ }^{d_{k}^{j}}=\left\langle f, \psi_{j k}^{p}\right\rangle$. In applications, it is possible to distinguish $f(x)$ by the $j$ level of wavelet features. If the top level of ${ }^{\left\{s_{k}^{J+1}\right\}}$ is determinate, the lower levels of ${ }^{\left\{s_{k}\right\}}$ are got by using fast arithmetic. The simple algorithm of ${ }^{\left\{s_{k}\right\}}$ is as below.

$$
\left\langle f, \psi_{j k}^{p}\right\rangle=\sum_{-\infty}^{+\infty} 2^{-j / 2} \hat{\varphi}\left[\frac{2 n \pi}{2^{j}}\right] e^{-2 i n k \pi / 2} \hat{f}(n)
$$


The value of $\bar{\varphi}\left[\frac{2 n \pi}{2^{j}}\right]^{-2 i n k \pi / 2}$ can be pre-set. Herein, $\Omega=\left\{x_{n}, y_{n}\right\}$ and $\bar{\Omega}=\left\{\overline{x_{n}}, \overline{y_{n}}\right\}$ are two closed curves in the same plane, $\left\{d_{k}\right\}$ and $\left\{\bar{d}_{k}^{j}\right\}$ are the wavelet features of the feature function. According to comparison methods, the matching formula is as below.

$$
E(j)=1-\frac{\sqrt{\sum_{k=0}^{2^{j}-1}\left(d_{k}^{j}-\overline{d_{k}^{j}}\right)^{2}}}{\left.\sqrt{\sum_{k=0}^{2^{j-1}}\left(d_{k}^{j}\right)^{2}}+\sqrt{\sum_{k=0}^{2^{j-1}}\left(\overline{d_{k}^{j}}\right)^{2}}\right]}
$$

The range of $\left.{ }^{E j} j\right)$ is $[0,1]$. When $d_{k}^{j}=\bar{d}_{k}^{j}$, the value of ${ }^{E(j)}$ is 1 . If $d_{k}^{j}=-t \bar{d}_{k}^{j}(t>0)$, the value of $\left.{ }^{E j}\right)$ is 0 . For the difference between low and high frequency components, the matching effect is different. The matching formula is defined as below.

$$
M(J)=\frac{\left[\frac{1}{2}\left(\frac{\left|s_{0}^{0}-s_{0}^{-0}\right|}{\left(\left|s_{0}^{0}\right|+\left|s_{0}^{-0}\right|\right)}\right)+\sum_{j=0}^{J}(1 / 2)^{j+2} E(j)\right]}{\left(1-(1 / 2)^{J+2}\right)}
$$

Herein, $\bar{\Omega}$ is got from the transformations of $\Omega$. The transformations include translating, rotating and scaling. So $\mathrm{M}(\mathrm{J})=1(\mathrm{~J}=0,1,2, \ldots)$.

\section{Construction of Compactly Supported Biorthogonal Wavelet}

The Compactly Supported Biorthogonal Wavelet is built to resolve the matching problem of feature regions. The frequency domains of biorthogonal scale function and wavelet function are as below $[3,4]$.

$$
\left\{\begin{array}{l}
\Phi(\omega)=H\left(\frac{\omega}{2}\right) \Phi\left(\frac{\omega}{2}\right) \\
\Psi(\omega)=G\left(\frac{\omega}{2}\right) \Phi\left(\frac{\omega}{2}\right) \\
\tilde{\Phi}(\omega)=\tilde{H}\left(\frac{\omega}{2}\right) \tilde{\Phi}\left(\frac{\omega}{2}\right) \\
\tilde{\Psi}(\omega)=\tilde{G}\left(\frac{\omega}{2}\right) \tilde{\Phi}\left(\frac{\omega}{2}\right)
\end{array}\right.
$$

If the biorthogonal scale functions such as $\varphi(t)$ and $\tilde{\varphi}(t)$ are defined as below, ${ }^{H(\omega)}$ and ${ }^{\tilde{H}(\omega)}$ are determined.

$$
\begin{aligned}
& G(\omega)=e^{-i \omega} \overline{\tilde{H}}(\omega+\pi) \\
& \tilde{G}(\omega)=e^{-i \omega} \bar{H}(\omega+\pi)
\end{aligned}
$$

Herein, ${ }^{H(\omega)}$ and $\tilde{H}(\omega)$ are as below.

$$
\begin{aligned}
& H(\omega)=\left(\cos \frac{\omega}{2}\right)^{2 K} Q(\cos \omega) \\
& \tilde{H}(\omega)=\left(\cos \frac{\omega}{2}\right)^{2 K} \tilde{Q}(\cos \omega)
\end{aligned}
$$




$$
\left\{\begin{array}{c}
L=K+\tilde{K} \\
\tilde{Q}(\cos \omega) Q(\cos \omega)=R\left(\sin ^{2} \frac{\omega}{2}\right) \\
R(x)=\sum_{j=0}^{L-1}\left(\begin{array}{c}
L-1+j \\
j
\end{array}\right) x^{j}
\end{array}\right.
$$

Herein, $K=1, \tilde{K}=3$ and $\tilde{Q}(\cos \omega) \equiv 1$. The expression of ${ }^{H(\omega)}$ and $\tilde{H}(\omega)$ are as below.

$$
\begin{gathered}
\left\{\begin{array}{c}
\tilde{H}(\omega)=(\cos \omega)^{2 \tilde{k}} \\
H(\omega)=\left(\cos \frac{\omega}{2}\right)^{2 k} \sum_{j=0}^{L-1}\left(\begin{array}{c}
L-1+j \\
j
\end{array}\right) \sin ^{2 j} \frac{\omega}{2}
\end{array}\right. \\
\Rightarrow\left\{\begin{array}{c}
\tilde{H}(\omega)=\left(\cos \frac{\omega}{2}\right)^{6} \\
H(\omega)=\left(\cos \frac{\omega}{2}\right)^{2} \sum_{j=0}^{3}\left(\begin{array}{c}
3+j \\
j
\end{array}\right) \sin ^{2 j}\left(\frac{\omega}{2}\right)
\end{array}\right.
\end{gathered}
$$

The resulting low-pass filters are shown in table 1. The high-pass filters are shown in table 2 and 3.[5]

Table 1 Low-pass filter $\tilde{h}_{n}$ and $h_{n}$

\begin{tabular}{|c|c|c|c|c|c|c|}
\hline & $n$ & 0 & \pm 1 & \pm 2 & & \pm 4 \\
\hline$\tilde{K}=3$ & $\tilde{h}_{n}$ & $\frac{5}{16} \sqrt{2}$ & $\frac{15}{64} \sqrt{2}$ & $\frac{3}{32} \sqrt{2}$ & $\frac{1}{64} \sqrt{2}$ & \\
$K=1$ & $h_{n}$ & $\frac{77}{32} \sqrt{2}$ & $-\frac{7}{32} \sqrt{2}$ & $-\frac{56}{64} \sqrt{2}$ & $\frac{15}{32} \sqrt{2}$ & $-\frac{5}{64} \sqrt{2}$ \\
\hline
\end{tabular}

Table 2 High-pass filter $g_{n}$

\begin{tabular}{|c|c|c|c|c|c|c|c|}
\hline$n$ & -2 & -1 & 0 & 1 & 2 & 3 & 4 \\
\hline$g_{n}$ & $-\frac{\sqrt{2}}{64}$ & $\frac{3 \sqrt{2}}{32}$ & $-\frac{15 \sqrt{2}}{64}$ & $\frac{5 \sqrt{2}}{64}$ & $-\frac{15 \sqrt{2}}{64}$ & $\frac{3 \sqrt{2}}{32}$ & $-\frac{\sqrt{2}}{64}$ \\
\hline
\end{tabular}

Table 3 High-pass filter

\begin{tabular}{|c|c|c|c|c|c|c|c|c|c|}
\hline$n$ & -3 & -3 & -1 & 0 & 1 & 2 & 3 & 4 & 5 \\
\hline & $-\frac{5 \sqrt{2}}{64}$ & $-\frac{15 \sqrt{2}}{32}$ & $-\frac{56 \sqrt{2}}{64}$ & $\frac{7 \sqrt{2}}{32}$ & $\frac{77 \sqrt{2}}{32}$ & $\frac{7 \sqrt{2}}{32}$ & $-\frac{56 \sqrt{2}}{64}$ & $-\frac{15 \sqrt{2}}{32}$ & $-\frac{5 \sqrt{2}}{64}$ \\
\hline
\end{tabular}

\section{Experiment of Feature Matching}

The scheme of repetitive confirmatory tests is designed that includes 100 times of back-and-forth movements carried out by a stepping motor. The stepping motor is controlled by the program that is developed for the research. Each movement includes 9 times of single-step movement and total number of steps is 900 times of single-step movement. The results show that the movement of the feature regions distribute from four to eight pixels. The cause of distribution is the limit of experimental conditions. The location precision of the platform used in the experiment is $0.05 \mathrm{~mm}$ and the repetitive positioning precision is $0.03 \mathrm{~mm}$. The image precision of $0.08 \mathrm{~mm}$ is very close to the precision of the platform. So the insufficient precision of the platform is the main reason of the distribution. Fig. 1 displays the data distribution of movements in pixels. It is clearly shown that the displacements symmetrically distribute around the mean value. The improvement of the precision of measurement system is needed to achieve micro-displacement measurement.

For all these reasons, the describing and matching approach could define the feature regions. The corresponding relation of movement between the CCD camera and the feature regions is built. Thus, the movement of the CCD camera could be calculated by the movement of the feature regions. 


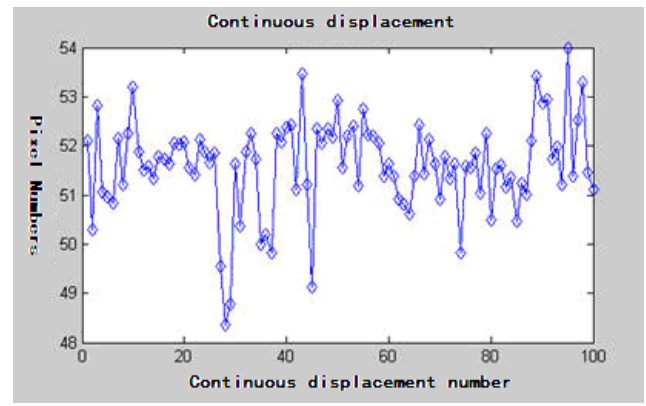

Fig.1 Experimental results

\section{Conclusion}

The paper presents Compactly Supported Biorthogonal Wavelet to describe and match the feature regions. The experiments clearly show that Compactly Supported Biorthogonal Wavelet can uniquely describe the edges of the feature regions. In the process of feature region description and matching, the relative displacement of feature regions is solved and the displacement of the CCD camera is also solved. Finally, it needs to be mentioned that the precision of displacement solution is insufficient. It is necessary to use high precision sub-pixel location technology to increase the measurement accuracy of the CCD camera.

\section{Acknowledgements}

The research work is supported by Shaanxi Provincial Special Fund (04JC11), President Fund of Xi'an Technological University (XGYXJJ0509) and Foundation of Key Subject Construction of Shaanxi Province

\section{References}

[1] TANG Bin-bing, CHEN Tuan-qiang, WANG Zheng-ming, Novel Image registration method based on wavelet transform. Journal of Computer Applications, 2007, 27(9) , P. 2103

[2] HU Shuo,ZHU Ming,WU Chuan,SONG Hua-jun, A Novel Starting-Point-independent Wavelet Coefficient Shape Matching. Journal of Image and Graphics, 2007, 12 (2), P.302

[3] SU Juan,QUAN Hong-yue, On the construction of bi-orthogonal Wavelet filter banks. Journal of Changsha Communications University, 2007, 27(3), P.78

[4] Fu Qinyi,Jiang Shuxia, Construction of a Compactly Supported Biorthogonal Wavelet. Journal of Vibration Engineering, 2004, 17(3), P.280

[5] XIA X G, Jaykuo C C,ZHANG Z. Wavelet coefficient computation with optimal profiteering. IEEE Trans Signal Processing. 1994,42(8) :2191-2197. 